\title{
3D PARALLEL SIMULATION MODEL OF CONTINUOUS BEAM ELECTRON CLOUD INTERACTIONS
}

\author{
A.Z. Ghalam, T. Katsouleas, University of Southern California, Los Angeles, California, USA \\ V.K. Decyk, C.K. Huang, W.B. Mori, University of California at Los Angeles, Los Angeles, \\ California, USA \\ G. Rumolo, GSI, Darmstadt, Germany \\ E. Benedetto, F. Zimmermann, CERN, Geneva, Switzerland
}

\begin{abstract}
Long-term beam-electron cloud interaction is modeled with a 3D parallel continuous model originally developed for plasma wakefield acceleration modeling. The simulation results are compared with the two macroparticle model for strong head-tail instability. The two macro- particle model qualitatively captures some of the instability features of the beam. The code is then used to model and make predictions for the beam dynamics in the presence of an electron cloud for several present and future circular machines around the world.
\end{abstract}

\section{INTRODUCTION}

Due to the importance of the electron cloud problem in many existing and planned circular machines around the world[1-9], many simulation codes have been developed to model the dynamics of the beam in the electron cloud. Most of these simulation codes such as HEAD-TAIL developed at CERN [10] are based on the single kick approximation where the cloud is lumped at discrete locations along the ring. While this approximation is very efficient as far as the computational costs are concerned, it has serious limitations [11]. The second simulation model recently developed at USC/UCLA is based on Particle-In-Cell method originally in use for plasma wakefields [12]. In this model the cloud is spread all over the ring and the beam continuously interacts with the electron cloud [12-15].

In this paper, QuickPIC as a more realistic model is used to model long-term beam- electron cloud interaction. This paper is organized as follows. In the second section some of the instability features of the beam are described with the two macro-particle model for strong head-tail instability. The last section is dedicated to the specific QuickPIC simulation results for SLAC-PEP-II and CERN-LHC storage ring.

\section{COMPARING THE SIMULATION RESULTS WITH THE TWO MACRO- PARTICLE MODEL}

In this section, some of the instability features of beamelectron cloud interaction observed in QuickPIC simulations are explained by the two macro-particle model for strong head-tail instability.
First, the validity of the instability criterion obtained in the two macro-particle modeling is investigated with QuickPIC simulations.

During the first half of the synchrotron period, $0<s / c<T_{s} / 2$, the equations of motion for the two macroparticles are:

$$
\begin{aligned}
& y_{1}^{\prime \prime}+\left(\frac{\omega_{\beta}}{c}\right)^{2} y_{1}=0 \\
& y_{2}^{\prime \prime}+\left(\frac{\omega_{\beta}}{c}\right)^{2} y_{2}=k y_{1}
\end{aligned}
$$

where $\omega_{\beta}$ is the betatron frequency of the particles in the transverse plane and $k$ is the coupling term between the two particles. In this case $k$ is proportional to transverse electron cloud wake produced at particle 2 caused by particle 1 . Similarly, during the second half period, the same equations apply with indices 1 and 2 exchanged. The right-hand side of the harmonic oscillator equation for the tail particle (index 2) corresponds to the force from the electron cloud wakefields generated due to the beam-electron cloud interaction. In writing these equations, only the first order wake function is considered for simplicity [11].

The stability condition for the center of charge motion dictates the following condition on the instability parameter, G:

$$
G=\frac{k \pi c^{2}}{2 \omega_{\beta} \omega_{s}}<2
$$

The instability parameter, $G$ depends on several parameters as shown in Eq.(2). In this section, the validity of the above criterion for beam stability in the presence of the electron cloud is investigated.

For the purpose of demonstration, the CERN-SPS parameters have been used in these simulations. These parameters are summarized in Table 1 of Appendix-A.

First Eq.(2) is used to find the instability threshold for the electron cloud density. The instability parameter $G$, can be specified once $k$ is determined. This coefficient can be found by QuickPIC simulations. To find the wake force at the tail, a point charge is set at the head of the 
bunch and the wake force is obtained at the location of the tail. Then the point charge is moved transversely and the wake force is again obtained at the tail. Repeating this process for different transverse offsets of the head, a plot of normalized wake force $\left(\frac{F_{\perp}}{2 m c^{2}}\right)$ versus transverse offset of the tail can be found as shown in Fig. 1.

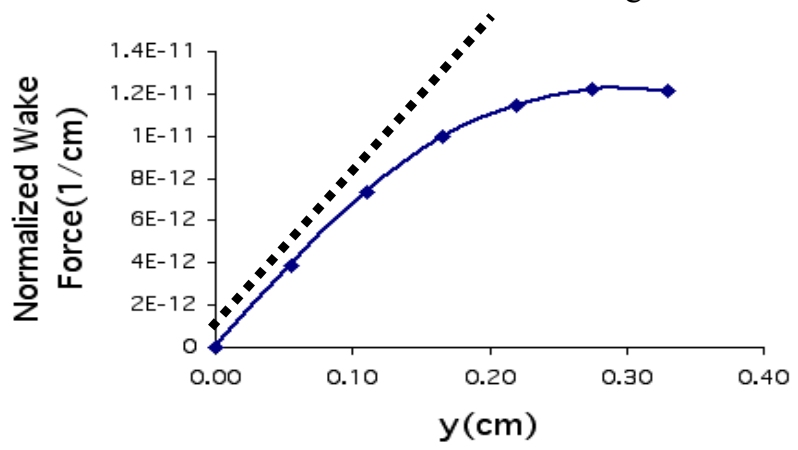

Figure 1: Normalized Wake force $\left(\frac{F_{\perp}}{2 m c^{2}}\right)$ at the tail of the bunch versus head offset in vertical plane

The rms spot size of the bunch for CERN-SPS is $0.2 \mathrm{~cm}$ and as can be seen from the plot, it lies in the non-linear region of the wakefield. The linearized wake force is shown as a dotted line the slope of which is $k$ in Eq. (2). It is worth mentioning that the linearized wake force obtained here matches surprisingly well with an approximate expression for the normalized wake force [17]:

$$
\frac{F \perp}{m m c^{2}}=\frac{4 \pi r_{0} \rho}{\gamma} y
$$

where $r_{0}$ is the classical radius of a proton, $\rho$ is the electron cloud density and $\gamma$ is the relativistic factor.

To examine the instability criterion in Eq. (2), we have to find a value of $k$ (or in other words a cloud density) for which the instability parameter, $G$, approaches 2 . Based on Eq. (2), the head-tail instability occurs at a cloud density of $2 \times 10^{6} / \mathrm{cm}^{3}$. Next, the threshold for instability is obtained by direct QuickPIC simulations. Based on QuickPIC simulations, the centroid motion starts growing exponentially at electron cloud densities around $10^{7} / \mathrm{cm}^{3}$. Thus, the instability criterion based on macro-particle modeling underestimated the threshold of instability.

\section{RESULTS FOR SPECIFIC CURRENT AND FUTURE MACHINES}

In this section, QuickPIC simulation results for SLACPEP-II storage ring and the CERN-LHC ring are given. The spot size growth of the beam is predicted and the instability threshold for electron cloud density is estimated.

\section{a) SLAC-PEP-II Lower Energy Ring (LER) Storage Ring}

The parameters used for PEP-II LER simulations are summarized in Table 2 in Appendix-A. As seen by the table, the box size dimension is $2 \mathrm{~cm} \times 1 \mathrm{~cm}$ however the PEP-II LER has a round cross section $4.5 \mathrm{~cm}$ of radius. The actual dimension of the pipe is much larger than the beam dimensions. Modeling the system with the actual pipe dimensions requires having many grids in the transverse plane in order to resolve the beam dimensions and this slows the simulation run time and makes it impractical to model long-term beam dynamics.

Since the pipe size is much larger than the beam spot size, the boundary conditions may be inconsequential to the long-term beam and electron cloud dynamics. To reduce the computational costs, the simulation box is reduced to the sizes indicated in Table 2. QuickPIC simulations confirms that the transverse wakefield in the reduced box size case differ from the original wakefields by less than $10 \%$.

Next, the long-term beam dynamics and instabilities have been analyzed for this ring. Figure 2 shows the horizontal spot size growth of the beam over 1000 turns of beam evolution around the ring for different electron cloud densities. As can be seen from the figure there is no growth for cloud densities of $5 \times 10^{5} / \mathrm{cm}^{3}$ and $10^{6} / \mathrm{cm}^{3}$. For density of $10^{7} / \mathrm{cm}^{3}$, the horizontal spot size grows steadily. Based on this simulation the cloud density threshold for horizontal beam blow-up is around $5 \times 10^{6} / \mathrm{cm}^{3}$.

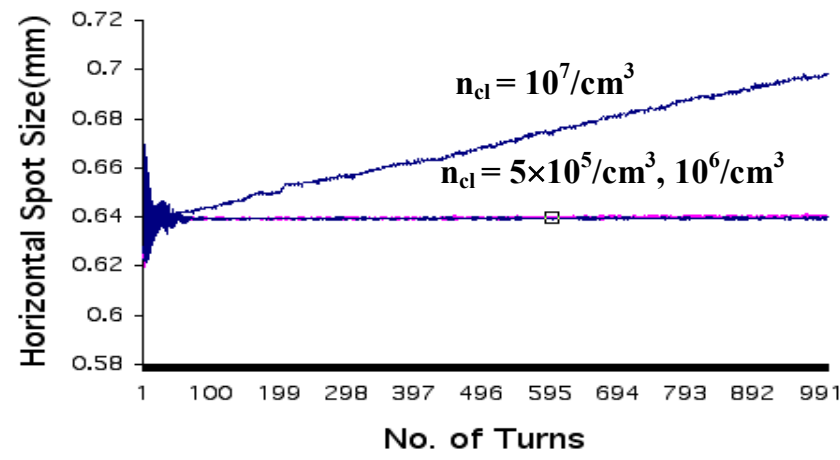

Figure 2: Horizontal spot size growth of the beam at PEPII LER ring for different cloud densities

Since the beam at PEP-II LER ring is flat, the dynamics of the beam in the vertical plane is expected to be different than that of the horizontal plane as illustrated in Figure 3. This figure shows the vertical spot size growth with different densities. 


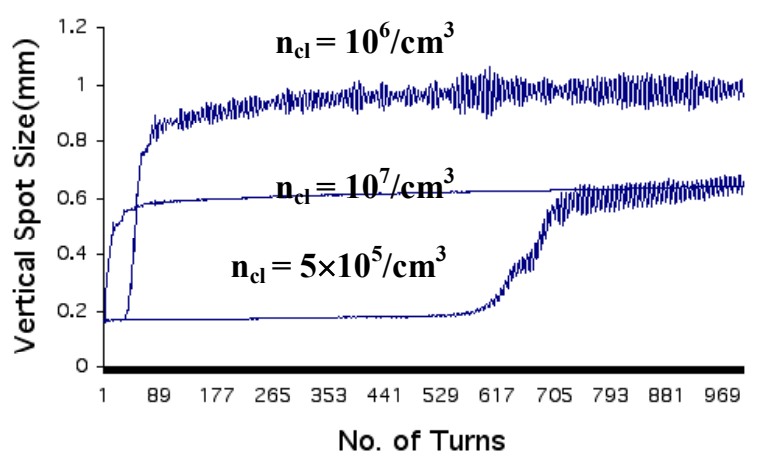

Figure 3: Vertical spot size of the beam at PEP-II LER with different cloud densities

For the cloud density of $10^{7} / \mathrm{cm}^{3}$, the spot size grows drastically in the first 100 turns and then the growth rate becomes much smaller. For the cloud densities of $10^{6}$ and $5 \times 10^{5} / \mathrm{cm}^{3}$, the situation is the opposite. There is almost no growth observed in the first turns and then the growth is drastic. While there is a consistent trend toward earlier growth the higher the cloud density, the saturated spot size does not appear to increase monotonically with cloud density. At this point we have no complete explanation for this non-monotonic behavior.

\section{b) CERN-LHC}

Next, the dynamics of the beam in the LHC ring is investigated with the QuickPIC simulations. The parameters for the simulation can be found in Table 3 in Appendix-A.

A typical Electron cloud density in this collider is predicted to be $6 \times 10^{5} \mathrm{~cm}^{-3}[16]$ and electron cloud would cause head-tail type instability in horizontal and vertical planes.

The spot size growth is investigated with QuickPIC simulations. Figure 4 shows the horizontal and vertical spot size growth for 2000 turns of beam evolution over the ring.

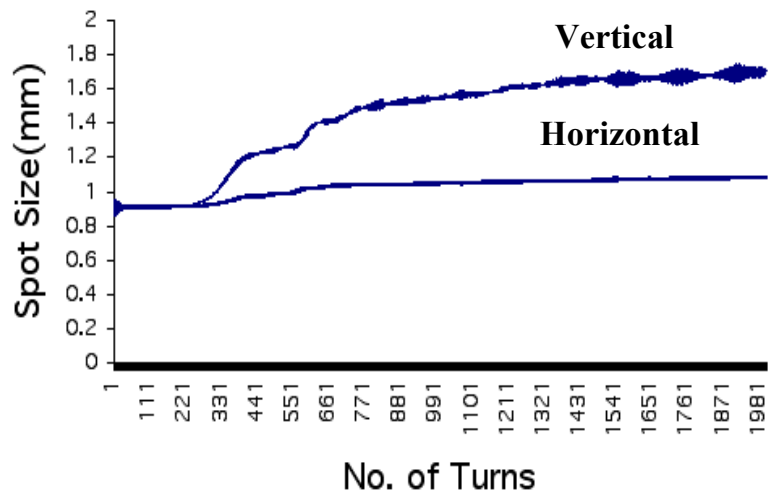

Figure 4: Horizontal and vertical spot size growth at CERN-LHC in a field free region.

\section{APPENDIX-A: TABLE OF PARAMETERS FOR DIFFERENT MACHINES}

Table 1: CERN-SPS parameters used in the simulations

\begin{tabular}{|l|c|}
\hline RMS Horizontal Spot Size $(\mathrm{mm})$ & 2 \\
\hline RMS Vertical Spot Size $(\mathrm{mm})$ & 2 \\
\hline RMS Bunch Length $(\mathrm{cm})$ & 30 \\
\hline Horizontal Box Size $(\mathrm{mm})$ & 80 \\
\hline Vertical Box Size $(\mathrm{mm})$ & 40 \\
\hline Bunch Population & $10^{11}$ \\
\hline Horizontal Emittance $(\mu \mathrm{m})$ & 0.1 \\
\hline Vertical Emittance $(\mu \mathrm{m})$ & 0.1 \\
\hline Momentum Spread & $2.48 \mathrm{E}-3$ \\
\hline Beam Momentum $(\mathrm{GeV} / \mathrm{c})$ & 26 \\
\hline Circumference $(\mathrm{km})$ & 6.9 \\
\hline Horizontal Betatron Tune & 26.22 \\
\hline Vertical Betatron Tune & 26.18 \\
\hline Synchrotron Tune & 0.005 \\
\hline Electron Cloud Density $\left(\mathrm{cm}^{-3}\right)$ & $10^{6}-10^{7}$ \\
\hline Number of Grids & $128 \times 64 \times 64$ \\
\hline Number of Beam Particles & 1048576 \\
\hline Number of Electron cloud Particles & 16384 \\
\hline
\end{tabular}

Table 2: SLAC-PEP-II Parameters used for the simulations

\begin{tabular}{|l|c|}
\hline RMS Horizontal Spot Size $(\mathrm{mm})$ & 0.623 \\
\hline RMS Vertical Spot Size $(\mathrm{mm})$ & 0.164 \\
\hline RMS Bunch Length $(\mathrm{cm})$ & 1.3 \\
\hline Horizontal Box Size $(\mathrm{mm})$ & 20 \\
\hline Vertical Box Size $(\mathrm{mm})$ & 10 \\
\hline Bunch Population & $10^{11}$ \\
\hline Horizontal Emittance $(\mu \mathrm{m})$ & 141 \\
\hline Vertical Emittance $(\mu \mathrm{m})$ & 8.806 \\
\hline Momentum Spread & $7.7 \times 10^{-4}$ \\
\hline Beam Momentum $(\mathrm{GeV} / \mathrm{c})$ & 3.19 \\
\hline Circumference $(\mathrm{m})$ & 2200 \\
\hline Horizontal Betatron Tune & 21.649 \\
\hline Vertical Betatron Tune & 19.564 \\
\hline Synchrotron Tune & 0.025 \\
\hline Electron Cloud Density $\left(\mathrm{cm}^{-3}\right)$ & $2 \times 10^{5}$ \\
\hline Number of Grids & $256 \times 128 \times 64$ \\
\hline Number of Beam Particles & 1048576 \\
\hline Number of Electron cloud Particles & 36864 \\
\hline
\end{tabular}


Table 3: CERN-LHC parameters used in the simulations

\begin{tabular}{|l|c|}
\hline RMS Horizontal Spot Size $(\mathrm{mm})$ & 0.884 \\
\hline RMS Vertical Spot Size $(\mathrm{mm})$ & 0.884 \\
\hline RMS Bunch Length $(\mathrm{cm})$ & 11.5 \\
\hline Horizontal Box Size $(\mathrm{mm})$ & 18 \\
\hline Vertical Box Size $(\mathrm{mm})$ & 18 \\
\hline Bunch Population & $1.1 \times 10^{11}$ \\
\hline Average Horizontal Beta Function & 66 \\
\hline Average Vertical Beta Function & 77.5 \\
\hline Momentum Spread & $4.68 \times 10^{-4}$ \\
\hline Beam Momentum $(\mathrm{GeV} / \mathrm{c})$ & 450 \\
\hline Circumference $(\mathrm{km})$ & 6.9 \\
\hline Horizontal Betatron Tune & 64.28 \\
\hline Vertical Betatron Tune & 59.31 \\
\hline Synchrotron Tune & 0.0059 \\
\hline Electron Cloud Density (cm $\left.{ }^{-3}\right)$ & $6 \times 10^{5}$ \\
\hline Number of Grids & $64 \times 64 \times 64$ \\
\hline Number of Beam Particles & 524288 \\
\hline Number of Electron cloud Particles & 16384 \\
\hline
\end{tabular}

\section{REFERENCES}

[1] H. Fukuma, K. Cornelis, F.-J. Decker, R. Macek, E. Metral, W. Fischer in Proceedings of ECLOUD'02, CERN, Geneva 15-18 April 2002, edited by G. Rumolo and F. Zimmermann, Yellow Report CERN-2002-001

[2] G. Rumolo, F. Ruggiero and F. Zimmermann, Phys. Rev. ST Accel. Beams, 4, 012801 (2001).

[3] M. Jimenez et al., in Proceedings of ECLOUD'02, CERN, Geneva 15-18 April 2002, edited by G. Rumolo and F. Zimmermann, Yellow Report CERN-2002-001

[4] A. Rossi, G. Rumolo, and F. Zimmermann, in Proceedings of ECLOUD'02, CERN, Geneva 15-18 April 2002, edited by G. Rumolo and F. Zimmermann, Yellow Report CERN-2002-001

[5] G. Arduini, K. Cornelis, W. Hoefle, G. Rumolo, and F. Zimmermann, in Proceedings of PAC2001, Chicago (2001)
[6] K. Oide, "Observation and Cure of the Electron Cloud Effect at the KEKB Low Energy Ring", Chamonix XI, CERN SL-2001-003 (DI)

[7] S. S. Win et al., "Observation of Transverse Coupled Bunch Instability at KEKB", in Proceedings of APAC2001, Bejing (2001)

[8] R. Cappi, M. Giovannozzi, E. Metral, G. Rumolo, and F. Zimmermann, Phys. Rev. ST Accel. Beams 5, 094401 (2002)

[9] K. Ohmi, "Particle in Cell Simulations of BeamElectron Cloud Interactions", PAC2001 Chicago.

[10] G. Rumolo et al., 'Electron Cloud Simulations: Beam Instabilities and Wakefields', in Proceedings of ECLOUD'02, CERN, Geneva, 2002.

[11] A. Chao ' Physics of Collective Beam Instabilities in High Energy Accelerators’ Wiley \& Sons 1993.

[12] A. Ghalam et. al., '3-D Parallel Simulation of Continuous Beam-Cloud Interactions', to be appeared in Proceedings of ECLOUD'04, Napa Valley, California

[13] E. Benedetto et. al.,'Simulation of Transverse Single Bunch Instabilities and Emittance Growth caused by Electron Cloud in LHC and SPS', to be appeared in Proceedings of ECLOUD'04, Napa Valley, California

[14] C. Hunag et. al., in proceedings of the $18^{\text {th }}$ Annual Review of Progress in Applied Electromagnetics, Monterey, CA, 2002, p.557

[15] G. Rumolo, A. Z. Ghalam et. al., Phys. Rev. ST Accel. Beams, Vol. 6, 081002, 2003.

[16] O. Bruning et al. (eds.), LHC Design Report Vol. 1: The LHC Main Ring, CERN-2004-003 (2004)

[17] K. Ohmi et. al., 'Head-Tail Instability Caused by Electron Clouds in Positron Storage Rings', PRL, Vol. 85, No. 18, 2000. 